\title{
L-type calcium current in right ventricular outflow tract myocytes of rabbit heart
}

\author{
LIANG ShengHui $^{1}$, LIN ChenHui $^{1}$, LI Yuan ${ }^{2}$, LIU TaiFeng $^{3} \&$ WANG Yan $^{1,2^{*}}$ \\ ${ }^{1}$ Fujian Medical University Graduate Student Education Institute, Fuzhou 350004, China; \\ ${ }^{2}$ Xiamen Heart Center, Xiamen Zhongshan Hospital of Xiamen University, Xiamen 361004, China, \\ ${ }^{3}$ Department of Physiology and Biophysics, College of Life Sciences, Peking University, Beijing 100871, China
}

Received September 28, 2011; accepted November 10, 2011

\begin{abstract}
The mechanism of idiopathic ventricular tachycardia originating from the right ventricular outflow tract (RVOT) is not clear. Many clinical reports have suggested a mechanism of triggered activity. However, there are few studies investigating this because of the technical difficulties associated with examining this theory. The L-type calcium current $\left(I_{\mathrm{Ca}-\mathrm{L}}\right)$, an important inward current of the action potential (AP), plays an important role in arrhythmogenesis. The aim of this study was to explore differences in the APs of right ventricular (RV) and RVOT cardiomyocytes, and differences in electrophysiological characteristics of the $I_{\mathrm{Ca}-\mathrm{L}}$ in these myocytes. Rabbit RVOT and RV myocytes were isolated and their AP and $I_{\mathrm{Ca}-\mathrm{L}}$ were investigated using the patch-clamp technique. RVOT cardiomyocytes had a wider range of AP duration (APD) than RV cardiomyocytes, with some markedly prolonged APDs and markedly shortened APDs. The markedly shortened APDs in RVOT myocytes were abolished by treatment with 4-AP, an inhibitor of the transient outward potassium current, but the markedly prolonged APDs remained, with some myocytes with a long AP plateau not repolarizing to resting potential. In addition, early afterdepolarization (EAD) and second plateau responses were seen in RVOT myocytes but not in RV myocytes. RVOT myocytes had a higher current density for $I_{\text {Ca-L }}$ than RV myocytes (RVOT $\left.(13.16 \pm 0.87) \mathrm{pA} \mathrm{pF}^{-1}, \mathrm{RV}(8.59 \pm 1.97) \mathrm{pA} \mathrm{pF}^{-1} ; P<0.05\right)$. The $I_{\mathrm{Ca}-\mathrm{L}}$ and the prolonged APD were reduced, and the EAD and second plateau response disappeared, after treatment with nifedipine (10 $\mu$ mol L ${ }^{-1}$ ), which blocks the $I_{\text {Ca-L }}$. In conclusion, there was a wider range of APDs in RVOT myocytes than in RV myocytes, which is one of the basic factors involved in arrhythmogenesis. The higher current density for $I_{\mathrm{Ca}-\mathrm{L}}$ is one of the factors causing prolongation of the APD in RVOT myocytes. The combination of EAD with prolonged APD may be one of the mechanisms of RVOT-VT generation.
\end{abstract}

arrhythmogenesis, cardiomyocytes, ventricular tachycardia, right ventricular outflow tract, L-type calcium current, triggered activity, early afterdepolarization, patch-clamp technique

Citation: $\quad$ Liang S H, Lin C H, Li Y, et al. L-type calcium current in right ventricular outflow tract myocytes of rabbit heart. Sci China Life Sci, 2012, 55: 41-46, doi: 10.1007/s11427-012-4265-3

Idiopathic ventricular tachycardia (IVT) refers to ventricular tachycardia in patients with no organic heart disease or other factors which cause cardiac arrhythmia [1-3]. 70\%-80\% of IVT originates from the right ventricular outflow tract (RVOT) and is called RVOT ventricular tachycardia (RVOT-VT) [1]. Embryological studies have shown that the RVOT and RV cardiomyocytes have different origins, with

*Corresponding author (email: wy@ medmail.com.cn)
RVOT cells originating from the bulbus arteriosus [4-6]. As IVT most commonly originates from the RVOT, it is important to study differences between the electrophysiological characteristics of RVOT and RV myocytes. Because of technical limitations, such as the difficulty in distinguishing morphologically between these myocyte types, there have been few experimental studies examining these differences, even though many clinical reports have speculated on the mechanisms underlying RVOT-VT $[7,8]$. The primary is- 
sues to be determined are the characteristics of RVOT myocyte functions, and the differences between RVOT and RV myocyte functions. This study examined the characteristics of the action potential (AP) and L-type calcium current $\left(I_{\mathrm{Ca}-\mathrm{L}}\right)$ in RVOT and RV myocytes.

A preliminary report (abstract) of this paper has previously been published [9].

\section{Materials and methods}

\subsection{Composition of solutions}

Bath solution without calcium consisted of $\left(\mathrm{mmol} \mathrm{L}^{-1}\right)$ : $\mathrm{NaCl}$ 137, $\mathrm{KCl} 5.4, \mathrm{MgCl}_{2}$ 1.0, $\mathrm{NaH}_{2} \mathrm{PO}_{4}$ 0.33, HEPES 5, glucose 10; pH 7.35-7.38.

$\mathrm{KB}$ solution consisted of $\left(\mathrm{mmol} \mathrm{L} \mathrm{L}^{-1}\right): \mathrm{KCl} 30$, L-Glutamic acid 50, taurine 20, $\mathrm{KH}_{2} \mathrm{PO}_{4} 30, \mathrm{MgCl}_{2}$, HEPES 10, glucose 10, EGTA 0.5; pH 7.38.

Extracellular solution for AP recording consisted of

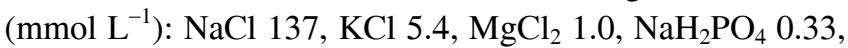
HEPES 5, glucose 10, $\mathrm{CaCl}_{2}$ 1.8; $\mathrm{pH}$ 7.35-7.38.

Pipette solution for AP recording consisted of (mmol $\mathrm{L}^{-1}$ ): $\mathrm{KCl} 150, \mathrm{MgCl}_{2}$ 1, HEPES 5, EGTA 5, $\mathrm{K}_{2}$-ATP 3, Mg-ATP 1; pH 7.3.

Extracellular solution for $I_{\mathrm{Ca}-\mathrm{L}}$ measurement consisted of (mmol L ${ }^{-1}$ ): $\mathrm{NaCl} 137, \mathrm{KCl} 5.4, \mathrm{MgCl}_{2}$ 1.0, $\mathrm{NaH}_{2} \mathrm{PO}_{4}$ 0.33, HEPES 5, glucose 10, $\mathrm{CaCl}_{2}$ 1.8, 4-AP 5; pH 7.35-7.38.

Pipette solution for $I_{\mathrm{Ca}-\mathrm{L}}$ measurement consisted of $\left(\mathrm{mmol} \mathrm{L}{ }^{-1}\right): \mathrm{KCl} 150, \mathrm{MgCl}_{2}$ 1, HEPES 5, EGTA 5, $\mathrm{K}_{2}$-ATP 3, Mg-ATP 1; pH 7.3.

\subsection{Rabbit ventricular myocytes}

Male New Zealand rabbits (obtained from Xiamen University Animal Center) weighing $1.5-2.5 \mathrm{~kg}$ were used. The animals were anesthetized, and the heart was rapidly excised and dropped into a beaker of cold (on crushed ice, $\sim 2^{\circ} \mathrm{C}$ ) bath solution without calcium before being perfused with a modified Langendorff preparation. Heart isolation and initiation of perfusion were completed within 1-2 min. After 5-10 min of preliminary perfusion with calcium-free bath solution (to wash out the blood and stabilize the heart), the solution was equilibrated with $99 \% \quad \mathrm{O}_{2}$ and passed through the heart a single time without recirculation. Following another 10-15 min of perfusion with an enzyme solution (recirculated), muscle was harvested from the RVOT and from right the RV far from the RVOT. Harvested muscle tissues were chopped and kept in $\mathrm{KB}$ solution at $4^{\circ} \mathrm{C}$, and used within $1-8 \mathrm{~h}[10]$.

\subsection{Electrophysiological recordings [11]}

Cells were placed in a bath chamber and allowed to settle for $10 \mathrm{~min}$. The extracellular solution was superfused at a rate of $2 \mathrm{~mL} \mathrm{~min}{ }^{-1}$. Experiments were performed at 36- $37^{\circ} \mathrm{C}$ using rod-shaped, non-contracting myocytes with clear striations, and an EPC10 amplifier (Heka Electronic Co., Germany). Records were stored on a PC computer using Patch Master software (Heka Electronic Co., Germany). Micropipettes pulled from borosilicate glass by a pipette puller (Sutter, American) to obtain a resistance of 3-6 M $\Omega$. Series resistance compensation was $60 \%-90 \%$, and input capacitance was $60-140 \mathrm{pF}$.

$I_{\mathrm{Ca}-\mathrm{L}}$ and AP were recorded using the whole-cell patchclamp technique. APs were induced with the current-clamp technique (current pulses of $5 \mathrm{~ms}$ duration, $1 \mathrm{~s}$ interval, 900 pA intensity). To construct current-voltage ( $I-V)$ curves for L-type calcium channels, the cells were voltage-clamped at a holding potential of $-40 \mathrm{mV}$ and $I_{\mathrm{Ca}-\mathrm{L}}$ was generated by stepwise $10 \mathrm{mV}$ depolarizing pulses (500 ms duration, $1 \mathrm{~s}$ interval) with a constant holding potential of -40 to $+60 \mathrm{mV}$.

\subsection{Statistical analysis}

Results are expressed as mean \pm standard error. Multiple sample analysis of variance was used for statistical comparisons, with $N=$ number of myocytes. Differences were considered significant when $P<0.05$. SPSS16.0 software was used for analyses.

\section{Results}

\subsection{AP of RVOT and RV myocytes}

There were no significant differences between RVOT and RV myocytes when examined microscopically. However, there were obvious differences in electrophysiological properties between these two groups of cells, especially in the AP duration (APD). The range of APD in RV myocytes was relatively small, but in RVOT myocytes some APDs were very short, some were similar to those of RV myocytes, and some were very long (Figure 1).

Figure 1 shows the differences in APD between the two types of myocytes, which can be used as an index to recognize each type. Myocytes with APDs which were shorter or longer than APDs of RV myocytes were studied as typical RVOT myocytes.

Previous reports have suggested that the transient outward potassium current $\left(I_{\mathrm{To}}\right)$ is larger in RVOT myocytes than RV myocytes [12]. We blocked $I_{\text {To }}$ by 4-AP and observed the changes in APD in the two kinds of myocytes to determine the role of $I_{\mathrm{To}}$ in each.

Three types of AP were recorded in RV and RVOT myocytes (Figure 2). There were no significant differences in resting potential or maximum depolarization potential among these AP types.

The markedly shortened APDs of RVOT myocytes were abolished after treatment with 4-AP. It can therefore be speculated that $I_{\text {To }}$ plays an important role in the APD of RVOT myocytes. 
A

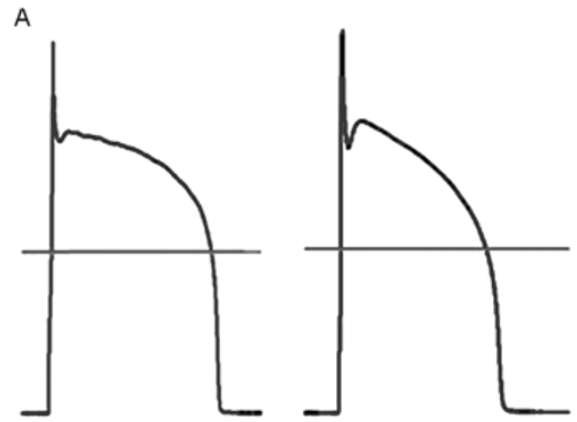

B

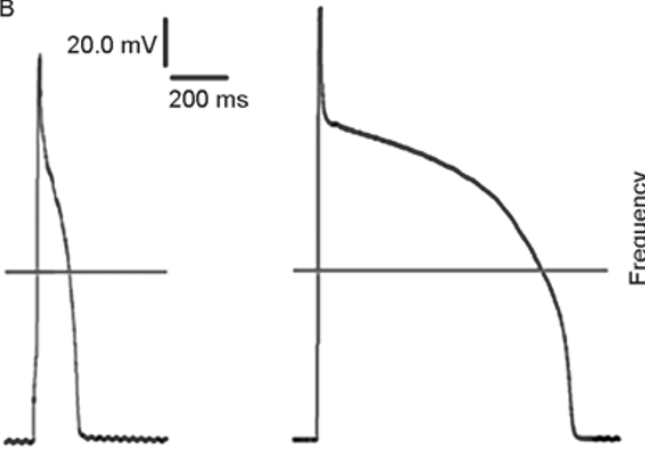

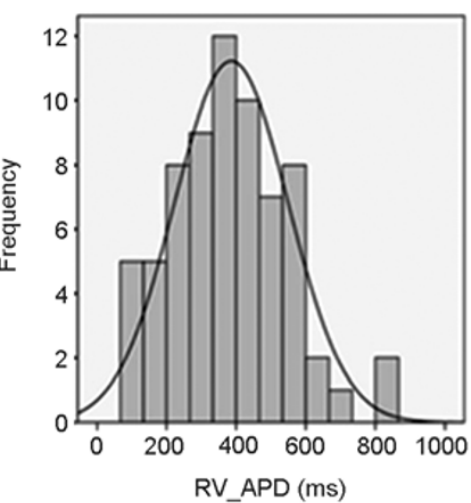

Mean $=386.2$

Std.Dev=163.4

$N=69$

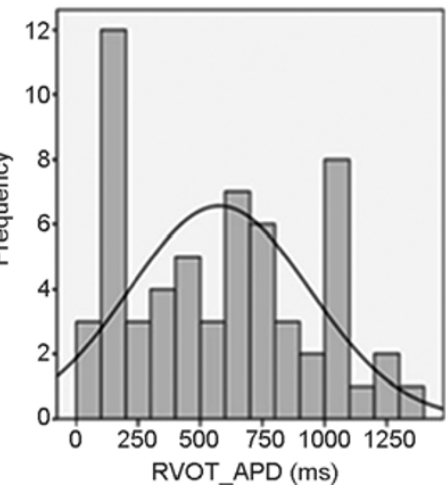

Mean $=577.2$

Std.Dev=384.8

$N=69$

Figure 1 APs of RVOT and RV myocytes. A, APs of RV myocytes. The frequency distribution histogram of APDs is shown on the right. APD=(386.2 \pm 163.4) ms, $N=69$. B, APs of RVOT myocytes. There was a wide range of APD. The shortest and longest APDs are shown. The frequency distribution histogram of APDs is shown on the right. APD $=(577.2 \pm 364.8) \mathrm{ms}, N=60$.
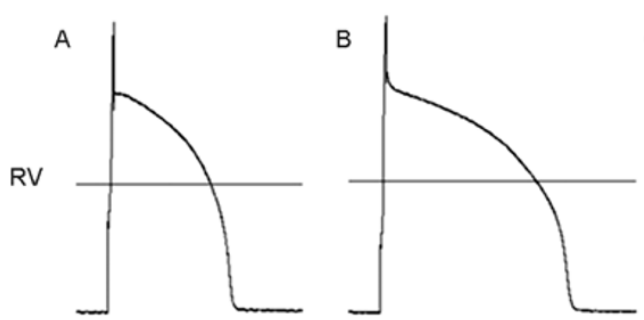

c
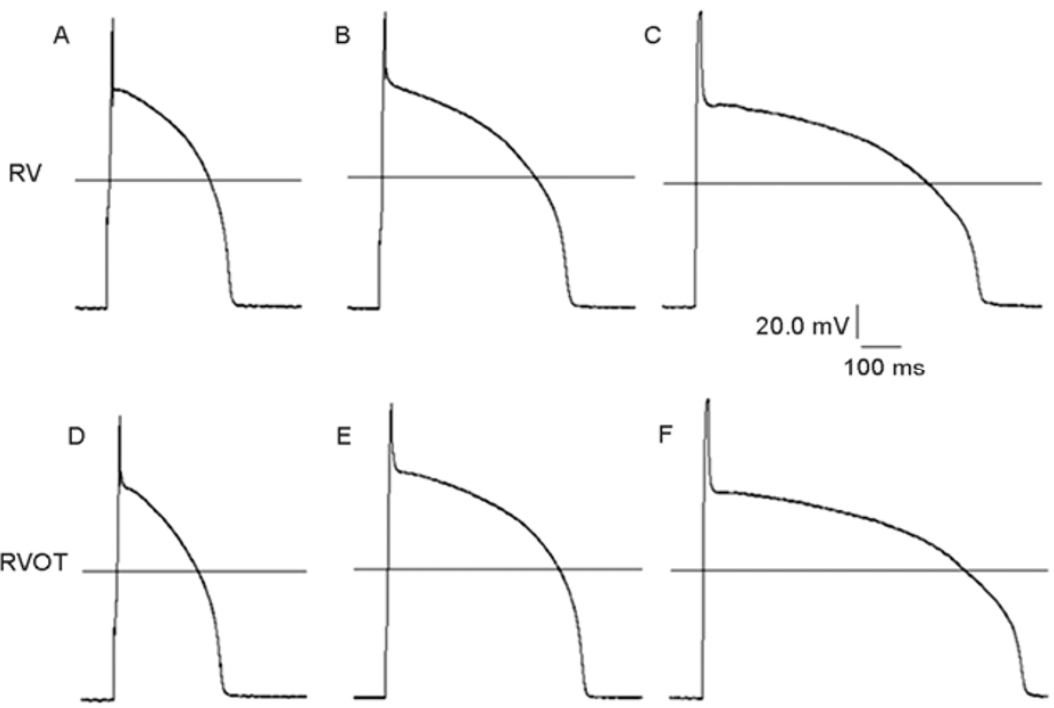

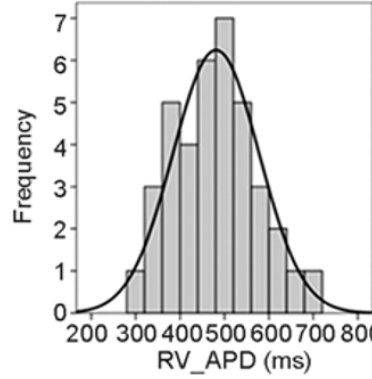

Mean $=481.0$ Std. Dev $=97.1$

$N=38$

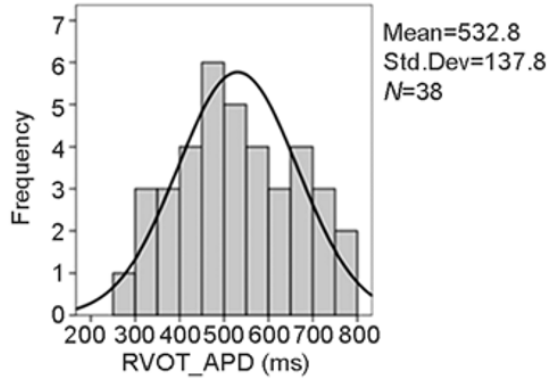

Figure 2 APs of RV and RVOT myocytes after 4-AP treatment (1 s interval). A, Short APD of RV myocytes. B, Intermediate APD of RV myocytes. C, Long APD of RV myocytes. D, Short APD of RVOT myocytes. E, Intermediate APD of RVOT myocytes. F, Long APD of RVOT myocytes. The frequency

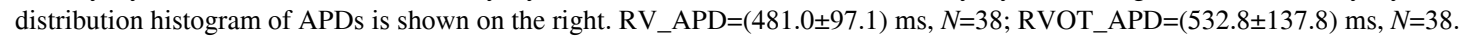

Without inducing factors, some RVOT myocytes with a long AP plateau did not repolarize to resting potential. EAD and a second plateau response [13] also occurred in some RVOT myocytes, but did not occur in RV myocytes (Figure 3).

\subsection{L-type calcium current}

After treatment with 4-AP, the cells were voltage-clamped at a holding potential of $-40 \mathrm{mV}$ to inactivate the sodium 
channel, and calcium currents were recorded at $0 \mathrm{mV}$. The APs of the RVOT and RV myocytes were positively correlated with $I_{\mathrm{Ca}-\mathrm{L}}$ density (Figure 4).

Using RV myocytes as the standard, the $95 \%$ reference range for APD (after $I_{\text {To }}$ was blocked) was calculated as 290-671 ms. If the APD of RVOT myocytes was within this range, they were categorized as normal-APD-RVOT myocytes. If the APD was not within this range, they were categorized as long-APD-RVOT or short-APD-RVOT myocytes. Differences among these groups were analyzed (Table 1).

Comparisons of differences in the mean values between groups using multiple sample analysis of variance did not identify significant differences between RV control myocytes and normal-APD-RVOT myocytes, but showed that the $I_{\text {Ca-L }}$ was larger in long-APD-RVOT myocytes than in RV control and normal-APD-RVOT myocytes.

Some RVOT myocytes with a very long AP plateau did not repolarize to resting potential in the testing interval. EAD and second plateau responses also occurred in these myocytes without inducing factors $(N=3)$ (Figure 3$)$. The $I_{\text {Ca-L }}$ was larger in these myocytes than in the RV control and normal-APD-RVOT myocytes. The $I$ - $V$ curves and active and inactive $I_{\mathrm{Ca}-\mathrm{L}}$ curves are shown in Figure 5.

The $I_{\mathrm{Ca}-\mathrm{L}}$ was reduced, the prolonged APD was shortened, and the EAD and second plateau response disappeared after treatment with nifedipine $\left(10 \mu \mathrm{mol} \mathrm{L}{ }^{-1}\right)$, a blocker of $I_{\mathrm{Ca}-\mathrm{L}}$ (Figure 6).

Comparison of the reactions of myocytes to treatment with nifedipine $\left(10 \mu \mathrm{mol} \mathrm{L}{ }^{-1}\right)$, which blocks L-type calcium channels, showed a larger reduction in APD in the longAPD-RVOT myocytes than in the RV control and normalAPD-RVOT myocytes. The APD remained longer in the long-APD-RVOT myocytes than in the RV control and the normal-APD-RVOT myocytes after treatment (Figure 7).

\section{Discussion}

There is currently no ideal method for identifying RVOT myocytes. This study presents a method of solving this dif-

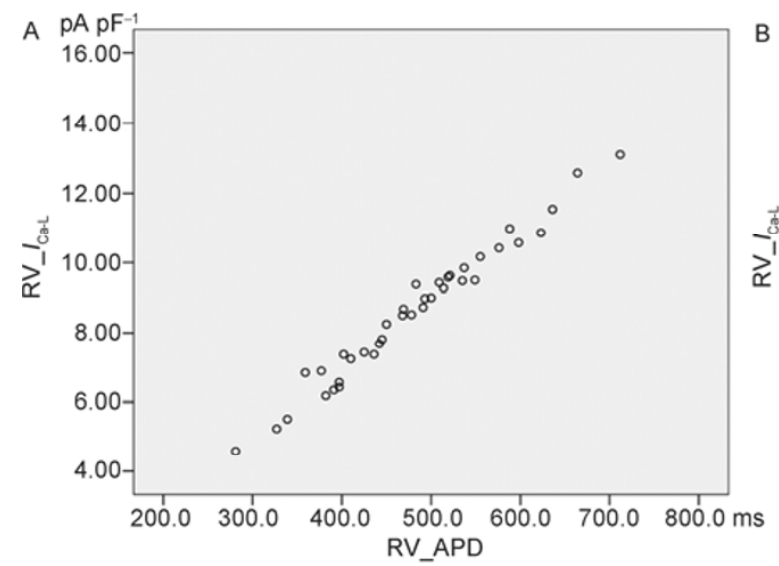

ficult problem, by measuring APD. The APD is related to stimulus frequency, but the change in APD depending on stimulus frequency is limited to a certain range, which is not lengthened or shortened arbitrarily. We used a fixed stimulus frequency to control for variables related to changes in frequency. Rabbit myocytes differ from those of other mammals in that the Kv1.4 protein expresses the $I_{\mathrm{To}}$ of the slow deactivation channel $\left(I_{\mathrm{To} . \mathrm{S}}\right)$. The response of rabbit myocytes to stimulus frequency is contrary to that of other mammals: when the stimulus frequency is fast, APD is lengthened, and when the stimulus frequency is slow, APD is shortened $[14,15]$. We observed this dependence of APD on stimulus frequency, and our results were consistent with those of previous studies [12], with APD lengthening as the stimulus frequency increased. We then observed the influence of 4-AP on this dependence, and found that 4-AP weakened the dependence of APD on stimulus frequency (Figure 8).

This experiment shows that the $I_{\text {To.S }}$ of rabbit myocytes influences APD, and that treatment with 4-AP reduces the effect of stimulus frequency on APD. This suggests that it is feasible to use APD as an index for RVOT myocyte identification.

Most authors believe that triggered activity is a possible

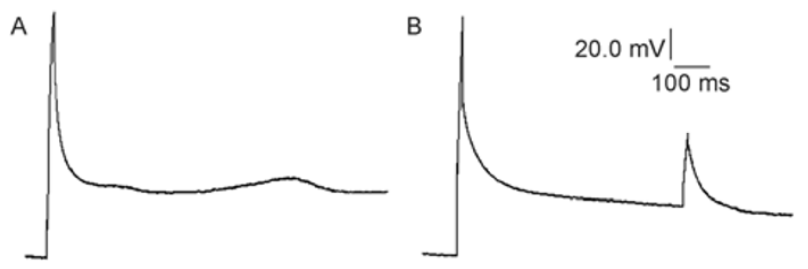

Figure 3 Triggered activity of RVOT myocytes (1 s interval). A, RVOT myocytes with a long AP plateau did not repolarize to resting potential, and the EAD generated spontaneously. B, Second plateau response in RVOT myocytes.

Table 1 Difference of $I_{\mathrm{Ca}-\mathrm{L}}$ in RV and RVOT myocytes

\begin{tabular}{cccc}
\hline Group & $N$ & $\mathrm{APD}(\mathrm{ms})$ & $I_{\mathrm{Ca}-\mathrm{L}}\left(\mathrm{pA} \mathrm{pF}{ }^{-1}\right)$ \\
\hline RV control myocytes & 38 & $481.0 \pm 97.12$ & $8.59 \pm 1.97$ \\
Normal-APD-RVOT myocytes & 29 & $488.28 \pm 96.46$ & $8.80 \pm 1.98$ \\
Long-APD-RVOT myocytes & 8 & $727.25 \pm 44.52$ & $13.16 \pm 0.87^{*}$ \\
Short-APD-RVOT myocytes & 1 & 271 & 4.83 \\
\hline
\end{tabular}

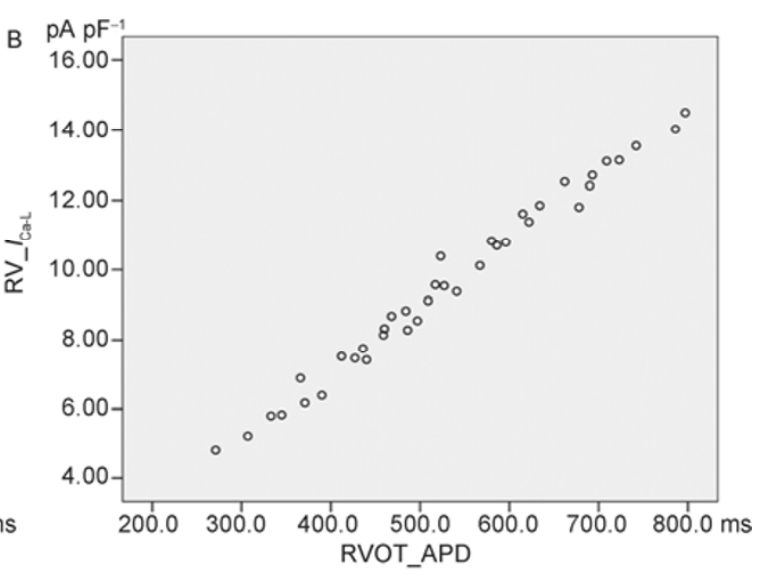

Figure 4 APs of RVOT and RV myocytes were positively correlated with $I_{\mathrm{Ca}-\mathrm{L}}$ density. 


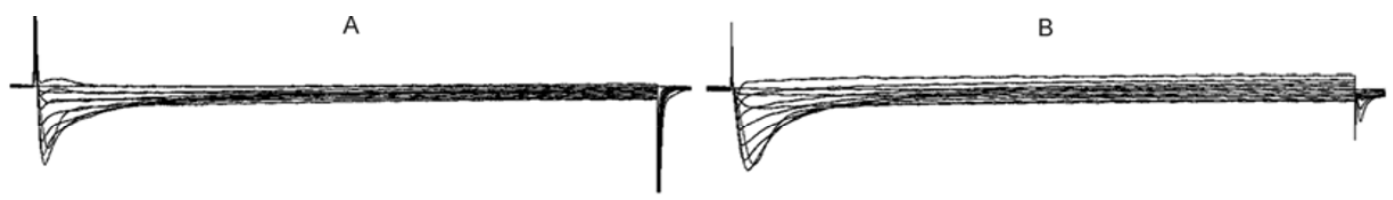

C
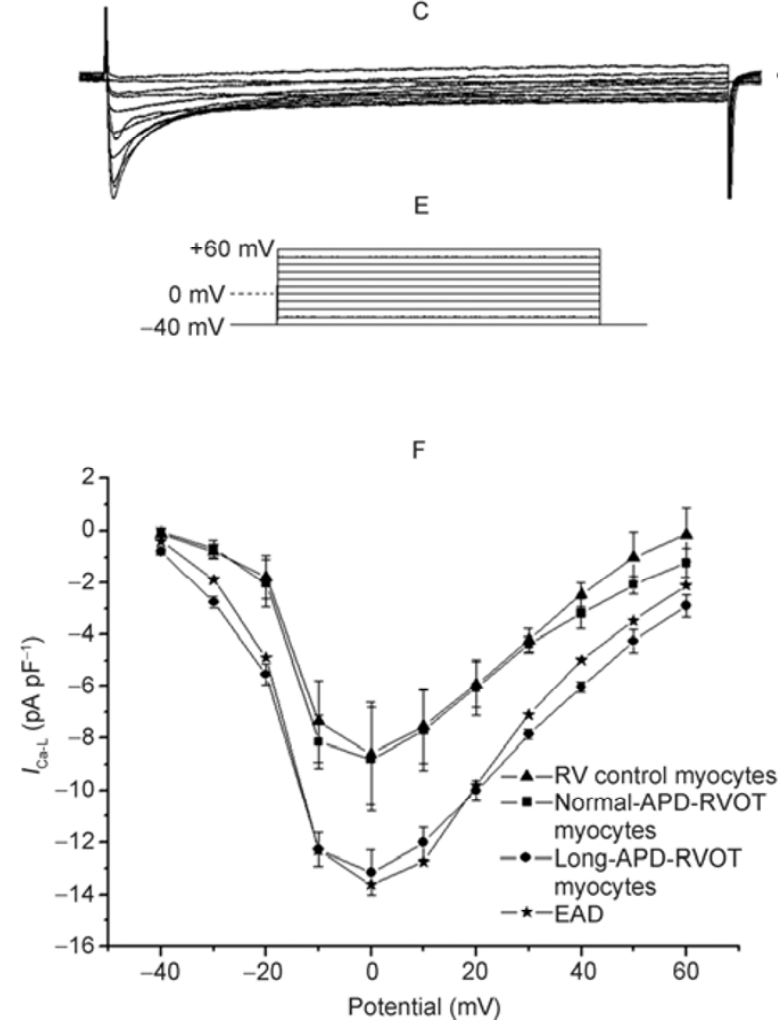

D

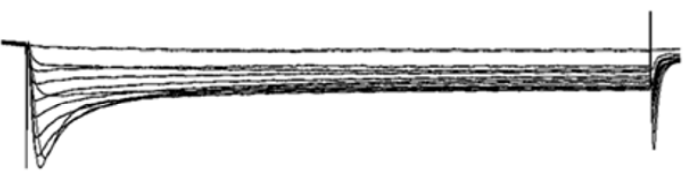

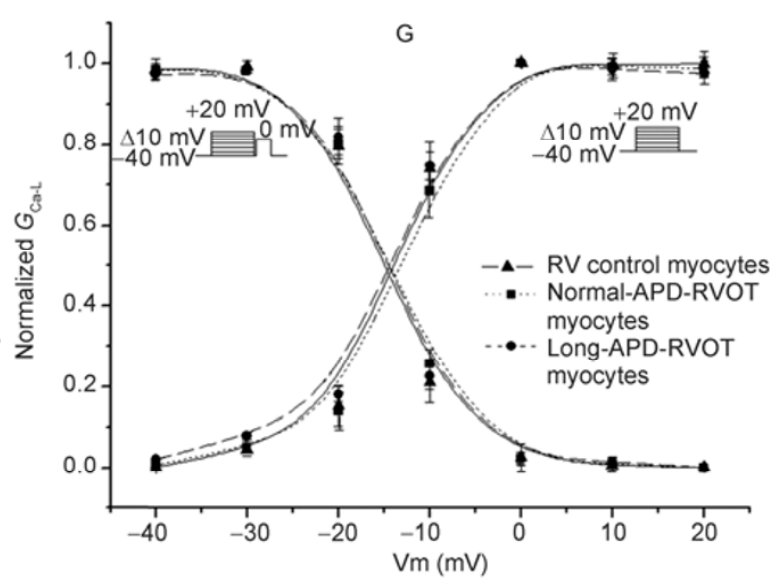

Figure $5 I_{\mathrm{Ca}-\mathrm{L}}$ of RV and RVOT myocytes. $I$ - $V$ curves, active and inactive $I_{\mathrm{Ca}-\mathrm{L}}$ curves. A, $I_{\mathrm{Ca}-\mathrm{L}}$ of RV control myocytes. B, $I_{\mathrm{Ca}-\mathrm{L}}$ of normal-APD-RVOT myocytes. C, $I_{\mathrm{Ca}-\mathrm{L}}$ of long-APD-RVOT myocytes. D, $I_{\mathrm{Ca}-\mathrm{L}}$ of myocytes in which EAD and a second plateau response were recorded. E, Protocol for $I_{\mathrm{Ca}-\mathrm{L}}$ generation (500 ms duration, $1 \mathrm{~s}$ interval). F, The $I$ - $V$ curve of long-APD-RVOT myocytes is significantly different from the $I$ - $V$ curves of RV control and normal-APD-RVOT myocytes. G, There were no significant differences in active and inactive $I_{\mathrm{Ca}-\mathrm{L}}$ curves among the three groups.
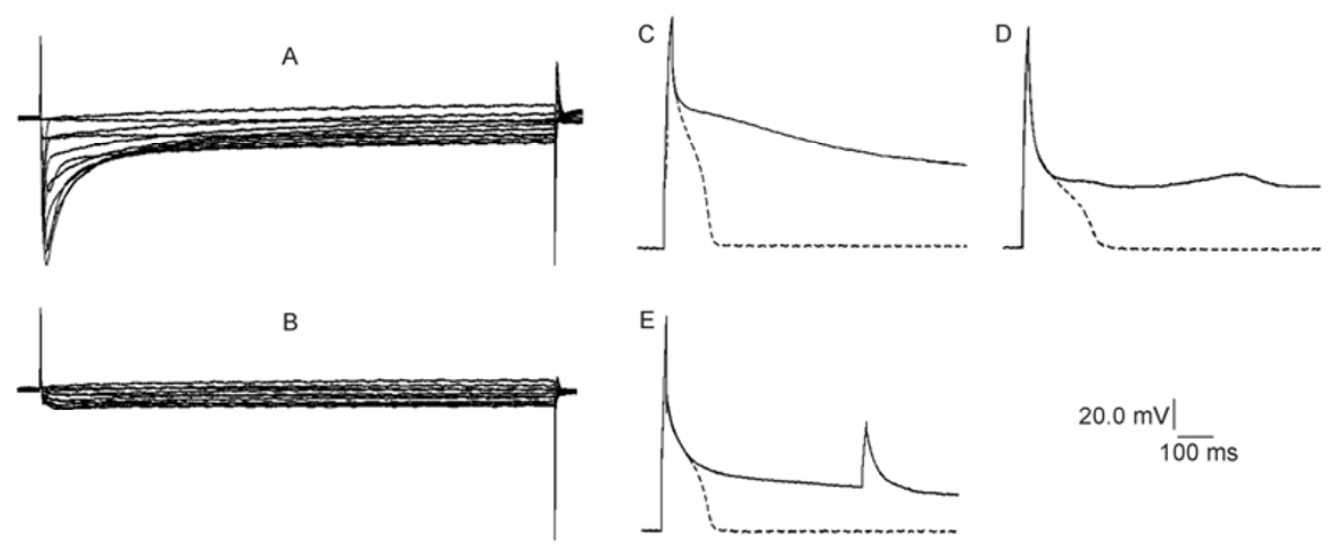

$20.0 \mathrm{mV}$

$1 \overline{00} \mathrm{~ms}$

Figure 6 A and B, The $I_{\mathrm{Ca}-\mathrm{L}}$ was reduced after treatment with nifedipine. C-E, The prolonged APD was shortened, and the EAD and second plateau response disappeared after treatment with nifedipine.

mechanism of RVOT-VT [16]. This study shows the possible mechanism of the triggered activity through observation of the AP and $I_{\mathrm{Ca}-\mathrm{L}}$ of RVOT myocytes.

In studying the mechanism of RVOT-VT, Song YanDong et al. [12] reported that a stronger $I_{\text {To }}$ increased APD dispersion in RVOT myocytes. In our experiment, we blocked the $I_{\mathrm{To}}$ with 4-AP to eliminate its influence on APD. We found that APD dispersion was reduced when the $I_{\mathrm{To}}$ was blocked, and that the short-APD-RVOT myocytes disappeared, indicating that the significant shortening of APD 


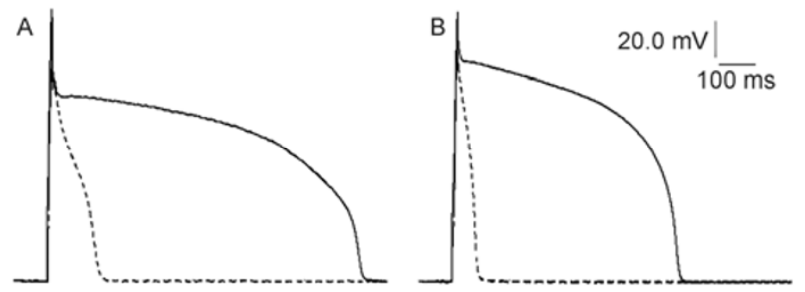

Figure 7 Reduction in APD after treatment with nifedipine $\left(10 \mu \mathrm{mol} \mathrm{L}{ }^{-1}\right)$. A, Long-APD-RVOT myocytes. B, RV control and normal-APD-RVOT myocytes. After treatment with nifedipine, there was a larger reduction in the APD of the long-APD-RVOT myocytes than in the RV control and

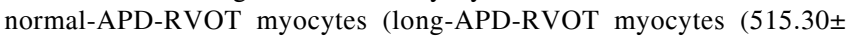
39.54) ms, RV control myocytes (369.49 \pm 65.44$) \mathrm{ms}$, normal-APD-RVOT myocytes $(380.28 \pm 50.58) \mathrm{ms})$. The APD remained longer in the long-APD-RVOT myocytes than in the RV control and normal-APD-

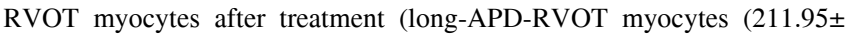
33.56) $\mathrm{ms}$, RV control myocytes $(111.51 \pm 43.21) \mathrm{ms}$, and normal-APDRVOT myocytes $(108 \pm 35.84) \mathrm{ms})$.

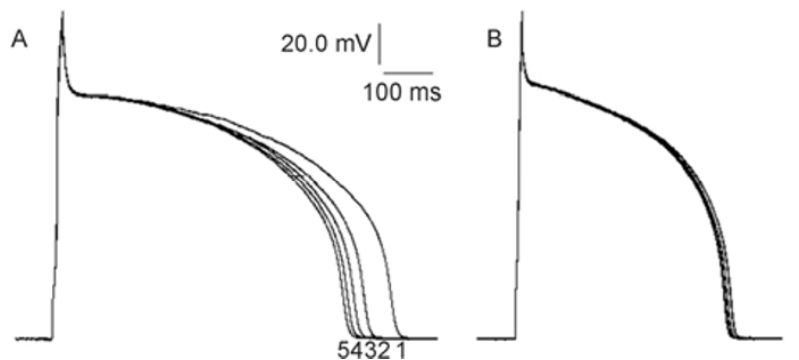

Figure 8 Dependence of APD on stimulus frequency. A, The APDs of $\mathrm{RV}$ and RVOT myocytes lengthened as the stimulus frequency increased. Stimulus frequency: $1,0.5 \mathrm{~s}$ internal; 2, $1 \mathrm{~s}$ internal; 3, $1.5 \mathrm{~s}$ internal; 4, $2 \mathrm{~s}$ internal; 5, $3 \mathrm{~s}$ internal). B, After treatment with 4-AP, the dependence of APD on stimulus frequency disappeared, and there were no significant differences in APD at different stimulus frequencies.

in RVOT myocytes might be because of a stronger $I_{\mathrm{To}}$, or amuch weaker sodium current, which is similar to Brugada syndrome type 1 .

Some RVOT myocytes had a long AP plateau which did not repolarize to resting potential within the testing period. EAD and a second plateau response were also seen in these myocytes. These cells showed a higher current density for $I_{\text {Ca-L }}$ than RV myocytes. After treatment with nifedipine, the $I_{\mathrm{Ca}-\mathrm{L}}$ and the APD were reduced, and the EAD and the second plateau response disappeared. This indicates a significant correlation between EAD and the $I_{\mathrm{Ca}-\mathrm{L}}$. There were no significant differences in the active and inactive $I_{\mathrm{Ca}-\mathrm{L}}$ curves among the groups, suggesting that the prolongation of APD was independent of the activation or inactivation of the channel.

The $I_{\text {Ca-L }}$ was obviously larger in the long-APD-RVOT myocytes than in the RV control and normal-APD-RVOT myocytes. After treatment with nifedipine, there was a larger reduction in APD in the long-APD-RVOT myocytes than in the RV control and normal-APD-RVOT myocytes, indi- cating that the $I_{\mathrm{Ca}-\mathrm{L}}$ played an important role in prolonging the APD. The APD remained longer in the long-APDRVOT myocytes than in the RV control and normal-APDRVOT myocytes after treatment with nifedipine, indicating that other channels in addition to L-type calcium channels also influence the prolongation of APD, such as the non-selective cationic channel. These results are consistent with the research by Song et al. [12].

In conclusion, RVOT myocytes had a wider range of APD than RV myocytes. This range is the basis of the short-QT and long-QT syndromes, and may also play a role in the mechanism of RVOT-VT. The $I_{\text {To }}$ could be one of the reasons for the significant shortening of the APD in RVOT myocytes. Myocytes with a significantly prolonged APD also had a higher current density for $I_{\mathrm{Ca}-\mathrm{L}}$, and were prone to EAD, which might induce RVOT-VT.

1 Altemose G T, Buxton A E. Idiopathic ventricular tachycardia. Cardiol Rev, 1999, 50: 159-177

2 Lerman B B, Stein K M, Markowitz S M. Mechanism of idiopathic left ventricular tachycardia. Cardiovasc Electrophysiol, 1997, 8: $571-583$

3 Lerman B B, Stein K M, Markowitz S M. Idiopathic ventricular outflow tract tachycardia: A clinical approach. Pacing Clin Electrophysiol, 1996, 19: 2120-2137

4 Moorman A F M, Christoffels V M. Cardiac chamber formation: development, genes, and evolution. Physiol Rev, 2003, 83: 1223-1267

5 Manner J. Ontogenetic development of the helical heart: concepts and facts. Europ J Cardiothor Surg, 2006: S69-S74

6 Chien K R, Domian I J, Parker K K. Cardiogenesis and the complex biology of regenerative cardiovascular medicine. Science, 2008, 322: 1494-1497

7 Boukens B J, Christoffels V M, Corinel R, et al. Developmental basis for electrophysiological heterogeneity in the ventricular and outflow tract myocardium as a substrate for life-threatening ventricular arrhythmias. Circ Res, 2009, 104: 19-31

8 Aliot E M, Stevenson W G, Almendral-Garrote J M, et al. EHRA/ HRS Expert consensus on catheter ablation of ventricular arrhythmias. Europace, 2009, 11: 771-817

9 Liang S H, Li Y, Liu T P. L-type calcium current of rabbit cardiomyocytes in right ventricular outflow tract. Heart, 2011, 97: A73

10 Chen J Q. Isolation techniqne of myocyte. Chin J Pathophysiol, 1999, 15: 475

11 Hamill O P, Marty A, Neher E, et al. Improved patch-clamp techniques for high-resolution current recording from ceils and cell-free membrane patches. Pflugers Arch, 1981, 391: 85

12 Song Y D, Yang X C, Liu T F, et al. The eletrophysiological basis of right ventricular outflow tract arrhythmogensis. Chin J Cardiac Pacing Electrophysiol, 2007, 21: 47-50

13 Liu T F, Han D Y. Role of take-off potential and second plateau response in generation of early afterdepolarization in atrial fibers of mouse heart. Meth Fin Exp Clin Pharmacol, 1991, 13: 181-185

14 Clark R B, Giles W R, Imaizumi Y. Property of the transient outward current in rabbit atrial cells. J Physiol, 1988, 405: 147-168

15 Fermini B, Wang Z, Duan D, et al. Differences in rate dependence of transient outward current in rabbit and human atrium. Am J Physiol, 1992, 263: H1747-1754

16 Lerman B B, Stein K M, Engelstein E D, et al. Mechanism of repetitive monomorphic ventricular tachycardia. Circulation, 1995, 93: 421-429

Open Access This article is distributed under the terms of the Creative Commons Attribution License which permits any use, distribution, and reproduction in any medium, provided the original author(s) and source are credited. 